\title{
CORRIGENDUM
}

\section{Impact of calibration on estimates of central blood pressures}

TK Soender, LM Van Bortel, JE Møller, J Lambrechtsen, J Hangaard and K Egstrup

Journal of Human Hypertension (2012) 26, 744; doi:10.1038/jhh.2012.31

Correction to: Journal of Human Hypertension (2012) 26, 706-710; doi:10.1038/jhh.2011.97; published online 3 November 2011

The authors of the manuscript JHH.2011.97 'Impact of calibration on estimates of central blood pressures' would like to clarify the follow-up period published in the manuscript.

Therefore, the last sentence in the introduction:

'The present study investigates whether the calibration method of the radial pressure wave changes estimates of central BPs and if it has an impact on the clinical interpretation of response to therapy in a population of patients with type II diabetes mellitus receiving intensified antihypertensive treatment for 6 months.'

has been changed to:

'The present study investigates whether the calibration method of the radial pressure wave changes estimates of central BPs and if it has an impact on the clinical interpretation of response to therapy in a population of patients with type II diabetes mellitus receiving intensified antihypertensive treatment.'
The sentences on the second page paragraph 3:

'Both clinic BP and ABPM were performed at baseline and after 6 months. During the 6 months patients with controlled hypertension $(\mathrm{CH})$ did not have any changes in their antihypertensive treatment performed by us and served as the control group.'

has been changed to:

'Both clinic BP and ABPM were performed at baseline and at the end of the follow-up period. During this follow-up period patients with controlled hypertension $(\mathrm{CH})$ did not have any changes in their antihypertensive treatment performed by us and served as the control group.'

Finally, the following sentence has been added as the first sentence in the Results section:

'Median follow-up time in the present study was 6 months with full and interquartile ranges of 2-18 months and 5-8 months, respectively.'

These errors have been rectified and the corrected article appears in this issue. The HTML and online pdf versions have also been rectified. 